\title{
Metformin Potentiates the Effects of Anlotinib in NSCLC via AMPK/mTOR and ROS-Mediated Signaling Pathways
}

\begin{abstract}
Zhongling $\mathrm{Zhu}^{1+}$, Teng Jiang ${ }^{1+}$, Huirong Suo ${ }^{2 \dagger}$, Shan $\mathrm{Xu}^{1}$, Cai Zhang ${ }^{1}$, Guoguang Ying ${ }^{3}$ and Zhao Yan ${ }^{1,4 *}$

${ }^{1}$ Department of Clinical Pharmacology, Tianjin Medical University Cancer Institute and Hospital, National Clinical Research Center for Cancer, Key Laboratory of Cancer Prevention and Therapy, Tianjin's Clinical Research Center for Cancer, Tianjin, China, ${ }^{2}$ Department of Pharmacy, The Second Hospital of Tianjin Medical University, Tianjin, China, ${ }^{3}$ Department of Tumor Cell Biology, Tianjin Medical University Cancer Institute and Hospital, National Clinical Research Center for Cancer, Key Laboratory of Cancer Prevention and Therapy, Tianjin's Clinical Research Center for Cancer, Tianjin, China, ${ }^{4}$ Department of Continuing Education and Science and Technology Service, China Anti-cancer Association, Tianjin, China
\end{abstract}

\section{OPEN ACCESS}

Edited by:

Aniello Cerrato,

Consiglio Nazionale Delle Ricerche

(CNR), Italy

Reviewed by:

Ganesh Prasad Mishra,

Swami Vivekanand Subharti University, India

Gamal Eldein Fathy

Abd-Ellatef Abd-Elrahman,

National Research Centre

(Egypt), Egypt

*Correspondence:

Zhao Yan

yanzhaopaper@163.com

${ }^{t}$ These authors have contributed equally to this work.

Specialty section:

This article was submitted to Pharmacology of Anti-Cancer Drugs, a section of the journal

Frontiers in Pharmacology

Received: 20 May 2021

Accepted: 21 July 2021

Published: 04 August 2021

Citation:

Zhu Z, Jiang T, Suo H, Xu S, Zhang C, Ying $G$ and Yan $Z$ (2021) Metformin Potentiates the Effects of Anlotinib in NSCLC via AMPK/mTOR and ROS-

Mediated Signaling Pathways.

Front. Pharmacol. 12:712181.

doi: 10.3389/fphar.2021.712181
Anlotinib is a novel multi-targeted tyrosine kinase inhibitor with activity against soft tissue sarcoma, small cell lung cancer, and non-small cell lung cancer (NSCLC). Potentiating the anticancer effect of anlotinib in combination strategies remains a clinical challenge. Metformin is an oral agent that is used as a first-line therapy for type 2 diabetes. Interesting, metformin also exerts broad anticancer effects through the activation of AMP-activated protein kinase (AMPK) and inhibition of mammalian target of rapamycin (mTOR). Here, we evaluated the possible synergistic effect of anlotinib and metformin in NSCLC cells. The results showed that metformin enhanced the antiproliferative effect of anlotinib. Moreover, anlotinib combined with metformin induced apoptosis and oxidative stress, which was associated with the activation of AMPK and inhibition of mTOR. Reactive oxygen species (ROS)- mediated p38/JNK MAPK and ERK signaling may be involved in the anticancer effects of this combination treatment. Our results show that metformin potentiates the efficacy of anlotinib in vivo by increasing the sensitivity of NSCLC cells to the drug. These data provide a potential rationale for the combination of anlotinib and metformin for the treatment of patients with NSCLC or other cancers.

Keywords: non-small cell lung cancer, anlotinib, metformin, AMP-activated protein kinase, reactive oxygen species

\section{INTRODUCTION}

Lung cancer is the leading cause of cancer-related death, accounting for over 1.7 million deaths per year worldwide (Bray et al., 2018). Approximately $85 \%$ of all lung cancers are non-small cell lung cancer (NSCLC). Most patients with NSCLC have advanced disease or local metastasis at diagnosis, and the 5 -years overall survival rate is less than $20 \%$ (Hirsch et al., 2017). In recent years, checkpoint inhibitors and inhibitors of constitutively active EGFR, ALK, or ROS1 receptor tyrosine kinases (RTKs) have markedly improved tumor responses and clinical outcomes in patients with NSCLC (Hirsch et al., 2017; Assi et al., 2018).

Anlotinib is a novel, multitargeted tyrosine kinase inhibitor that has activity against a range of RTKs involved in vascularization and tumor progression, including VEGFR-1, -2, and -3; FGFR-1, 
-2, -3, and -4; c-kit; and PDGFR- $\alpha$ and - $\beta$ (Shen et al., 2018; Gao et al., 2020). Several clinical trials have demonstrated that anlotinib is well tolerated and has promising efficacy in patients with solid tumors, including advanced NSCLC, soft tissue sarcoma, medullary thyroid carcinoma, esophageal squamous cell carcinoma, and metastatic renal cell carcinoma (Chi et al., 2018; Sun et al., 2018; Zhou et al., 2019; Ma et al., 2020; Wu et al., 2020; Huang et al., 2021). In phase III clinical trials, the median overall survival for patients with advanced NSCLC who progressed after treatment with at least two lines of prior systemic chemotherapy had increased by 3.3 months (Han et al., 2018). Based on these data, anlotinib was approved by the China National Medical Products Administration for third-line or further treatment of advanced NSCLC in 2018 (Syed, 2018).

The biguanide metformin is a first-line oral anti-diabetic drug. Several studies have shown that metformin inhibits cancer cell growth and induces both cell cycle arrest and apoptosis (Alimova et al., 2009; Dowling et al., 2011). Treatment with metformin has been reported to suppress the growth of tumor xenografts in nude mice (Wheaton et al., 2014). However, the mechanisms underlying these effects are poorly understood. It is known that metformin inhibits complex I of the mitochondrial electron transport chain (Fontaine, 2018), resulting in an increase in the intracellular AMP/ATP ratio and indirect activation of AMP-activated protein kinase (AMPK). AMPK activation promotes metabolic flexibility and net ATP conservation through multiple mechanisms, including activation of catabolic pathways, inhibition of anabolic processes that consume ATP, induction of autophagy, and maintenance of NADPH homeostasis to buffer reactive oxygen species (ROS). Retaining AMPK activity may protect tumor cells from bioenergetic catastrophe and provide them with a selective growth advantage under stress. Conversely, AMPK activation can inhibit mTOR signaling, leading to decreased HIFa-driven metabolism of glucose and glutamine (Faubert et al., 2015). Metformin can also exert anti-tumor effects through AMPKindependent pathways (Kalender et al., 2010).

Metformin was previously reported to increase the sensitivity of cancer cells to targeted therapies and chemotherapies (Zhang and Guo, 2016; Deng et al., 2019). Multiple combinations of metformin with targeted agents, such as gefitinib, trastuzumab, and temsirolimus, are currently being tested in phase I/II clinical trials (Khawaja et al., 2016; Zhang and Guo, 2016; Martin-Castillo et al., 2018). Here, we report the synergistic effect of anlotinib in combination with metformin in vitro and in vivo. We observed AMPK activation and inhibition of the downstream mTOR pathway, which may partly explain the synergistic cytotoxic effect. In addition, ROS-mediated p38/JNK MAPK and ERK signaling may be involved in the anticancer effect of the combination.

\section{MATERIALS AND METHODS}

\section{Reagents}

Anlotinib was kindly provided by Chia Tai Tian Qing Pharmaceutical Group Co., Ltd (Nanjing, China). Metformin,
3- (4,5-dimethylthiazol-2-yl)-2,5-diphenyltetrazolium bromide (MTT), methanol, crystal violet, and phosphate-buffered saline (PBS) were purchased from Solarbio Bioscience \& Technology Co. Ltd (Beijing, China). Hoechst 33342, propidium iodide (PI), 2,7-dichlorodihydrofluorescein diacetate (DCFH-DA), and SDS lysis buffer were obtained from Beyotime Biotechnology Co., Ltd (Shanghai, China). Dulbecco's modified Eagle's medium (DMEM), fetal bovine serum (FBS), trypsin, and penicillin/ streptomycin were purchased from Invitrogen (Carlsbad, CA, United States).

\section{Cell Culture}

The human lung cell lines A549 and H460 were obtained from American Type Culture Collection (Manassas, VA, United States) and were authenticated via DNA sequencing on an ABI 3730xl genetic analyzer. The cells were grown in DMEM supplemented with $10 \%$ heat-inactivated FBS, 100 units/ml penicillin, and $100 \mu \mathrm{g} / \mathrm{ml}$ streptomycin in a humidified atmosphere with $5 \%$ $\mathrm{CO}_{2}$ at $37^{\circ} \mathrm{C}$.

\section{MTT Cell Viability Assay}

Cells were seeded in 96-well culture plates at a density of 1-3 $\times$ $10^{3}$ cells/well. After $24 \mathrm{~h}$, various concentrations of anlotinib (range, $0-20 \mu \mathrm{mol} / \mathrm{L}$ ), metformin (range, $0-20 \mathrm{mmol} / \mathrm{L}$ ), or both were added to the cells. After $72 \mathrm{~h}$ of incubation, $5 \mathrm{mg} /$ $\mathrm{ml}$ MTT was added to each well and incubated for $4 \mathrm{~h}$. The supernatants were carefully aspirated and the formazan crystals were dissolved in DMSO. Absorbance was recorded at $570 \mathrm{~nm}$ using a microplate reader.

\section{Colony Formation Assay}

For clonogenic survival studies, 300 cells were seeded in 12-well plates and exposed to different treatments for $48 \mathrm{~h}$. After 10-14 days of incubation, the colonies were fixed in cold methanol for $6 \mathrm{~min}$ and then stained with $1 \%$ crystal violet solution for $30 \mathrm{~min}$. Colonies containing more than 50 cells were counted. Percent colony formation was calculated by comparison to that in untreated cultures, which was set to $100 \%$. Thus, the percent colony formation of treated cells was calculated as follows: colony formation by treated cells/colony formation by untreated cells $\times 100$.

\section{Apoptosis Assay}

The apoptosis assay was performed using Hoechst 33342/PI fluorescence double staining. Briefly, A549 and H460 cells were seeded at approximately $50 \%$ confluence in six-well cell culture plates. Thereafter, cells were incubated with anlotinib $(10 \mu \mathrm{M})$, metformin $(10 \mathrm{mM})$, or both for $24 \mathrm{~h}$. Finally, the treated cells were stained with Hoechst $33342(10 \mu \mathrm{g} / \mathrm{ml})$ and PI $(5 \mu \mathrm{g} / \mathrm{ml})$ at $37^{\circ} \mathrm{C}$ for $15 \mathrm{~min}$ and then imaged using fluorescence microscopy.

\section{Western Blot Analysis}

Cells were washed with ice-cold PBS and lysed with SDS lysis buffer. The protein concentration in the lysates was determined using BCA reagent (Pierce, Rockford, IL, United States). The proteins were separated via SDS-polyacrylamide gel 
electrophoresis and then electrotransferred onto PVDF membranes (Millipore, Bedford, MA, United States). The membranes were blocked with 5\% nonfat milk and incubated overnight at $4^{\circ} \mathrm{C}$ with primary antibodies against AMPK (\#5832), phospho-AMPK (Thr172) (\#2535), mTOR (\#2983), phosphomTOR (Ser2448) (\#5536), acetyl-CoA carboxylase (ACC) (\#3676), phospho-ACC (Ser79) (\#3661), hypoxia-inducible factor 1a (HIF1a) (\#36169), extracellular signal-regulated kinase (ERK1/2) (\#4695), phospho-ERK1/2 (Thr202/Tyr204) (\#4370), c-Jun NH 2-terminal kinase (JNK) (\#9252), phosphoJNK (Thr183/Tyr185) (\#4668), p38 (\#8690), phospho-p38 (\#4511) (Thr180/Tyr182), Bax (\#5023), Bcl-2(\#2870), cleaved caspase-3 (\#9664), and cleaved PARP (\#5625), which were purchased from Cell Signaling Technology (Danvers, MA, USA).Antibodies against $\beta$-actin (sc-47778) were purchased from Santa Cruz Biotechnology (Santa Cruz, CA, United States). After washing, the membranes were incubated with IRDye-conjugated anti-rabbit or anti-mouse IgG antibodies (LI-COR Biosciences, Lincoln, NE, United States). The proteins were visualized using an Odyssey LI-COR infrared imaging system.

\section{ROS Staining}

Intracellular hydrogen peroxide was detected using a DCFH-DA fluorescent probe. Cells were cultured in six-well plates and treated as indicated. Cells were washed twice with PBS and then incubated with $10 \mu \mathrm{M}$ DCFH-DA and $10 \mu \mathrm{g} / \mathrm{ml}$ Hoechst 33342 at $37^{\circ} \mathrm{C}$ for $30 \mathrm{~min}$ and then imaged using fluorescence microscopy.

\section{Determination of Intracellular ATP and the NADP $^{+} /$NADPH Ratio}

Relative intracellular ATP levels and the $\mathrm{NADP}^{+} / \mathrm{NADPH}$ ratio were determined using assay kits (Beyotime Biotechnology, Jiangsu, China) according to the manufacturer's instructions. Briefly, cells were cultured in six-well plates and treated as indicated. At harvest, the cells were washed twice with PBS and lysed in lysis buffer. After centrifugation at $12,000 \times g$ at $4^{\circ} \mathrm{C}$ for $5 \mathrm{~min}$, the supernatant was collected. Intracellular ATP levels were determined using a luminescent plate reader. The protein concentration was quantified using BCA reagent to normalize protein levels for calculating ATP content.

To determine the NADP ${ }^{+} / \mathrm{NADPH}$ ratio, the cells were washed twice with PBS and lysed in NADP extraction buffer. Following centrifugation at $12,000 \times g$ at $4^{\circ} \mathrm{C}$ for $10 \mathrm{~min}$, the supernatants were collected and analyzed to quantify the $\mathrm{NADP}^{+} / \mathrm{NADPH}$ ratio according to the manufacturer's instructions.

\section{Mouse Xenografts in vivo}

Four-week-old female BALB/c nude mice were purchased from Beijing HFK Bioscience Co., Ltd (Beijing, China). The animals were maintained under controlled environmental conditions: $22-28^{\circ} \mathrm{C}, 60-70 \%$ relative humidity, and a $12 \mathrm{~h}$ dark/light cycle with water ad libitum. A549 cells $\left(3 \times 10^{6}\right.$ cells) were intravenously injected into the left hind flanks of nude mice ( $\mathrm{n}=6$ mice per group). Tumor volume was calculated using formula $V=\left(a \times b^{2}\right) / 2$, where $a$ is the tumor length and $b$ is the tumor width. When the tumor volume reached approximately $100 \mathrm{~mm}^{3}$, the mice were randomly assigned to the control [treated with vehicle (sterile PBS)], anlotinib $(0.75 \mathrm{mg} / \mathrm{kg})$, metformin $(250 \mathrm{mg} / \mathrm{kg})$, or combination (anlotinib plus metformin) groups. Anlotinib and metformin were intragastrically administered daily for 28 consecutive days. Tumor growth was monitored and measured twice per week using a Vernier caliper. Tumors were removed from the mice after 28 days of treatment. The relative tumor volume (RTV) was calculated as the ratio of the tumor volume at time $t$ to the tumor volume at the start of treatment. Inhibition rates are expressed as the ratio of the RTV of the treatment group (TRTV) to the RTV of the control group (CRTV) by dividing the RTV of each treatment group by the RTV of the control group, and then multiplying the quotient by 100 (TRTV/CRTV\%). All protocols were approved by the Laboratory Animal Ethics Committee of Tianjin Medical University Cancer Institute and Hospital.

\section{Statistical Analysis}

In the xenograft experiment, randomization was performed using a computer-generated sequence of random numbers. In other experiments, randomization was not performed. Data are presented as the mean \pm SE from three independent experiments. Data were analyzed using GraphPad Prism 5.01. Mean values were compared using the unpaired Student's $t$-test. Normality distribution was assessed using the Shapiro-Wilk test. The combination index (CI) was calculated using CompuSyn software (Biosoft, Cambridge, United Kingdom). Statistical significance was set at $p<0.05\left(^{*}\right)$ or $p<0.01\left(^{* *}\right)$.

\section{RESULTS}

\section{Metformin Potentiates the Antiproliferative Effect of Anlotinib in NSCLC Cells}

To examine the potential synergistic effect of anlotinib and metformin on cancer cell proliferation, we studied this drug combination in the NSCLC cell lines A549 and H460 using MTT and colony formation assays. As shown in Figure 1A, although each agent alone inhibited the proliferation of A549 and $\mathrm{H} 46$ cells, the combination had the strongest antiproliferative effect. The CI values were calculated using cytotoxicity data from the MTT assay. The results revealed that the CI values were less than one in both cell lines (Figure 1B). A CI value $<1$ indicates drug synergism. The addition of metformin reduced the half-maximal inhibitory concentration of anlotinib by 2.7 -fold in A549 cells and by 4 . 0 -fold in H460 cells (Figure 1C). We also tested this combination in a 14-days colony formation assay. Similarly, combined treatment with anlotinib and metformin synergistically suppressed colony formation in both A549 and H460 cells (Figure 1D). These data indicate that the antiproliferative effects of anlotinib and metformin are strongly synergistic in A549 and H460 cells. 
A
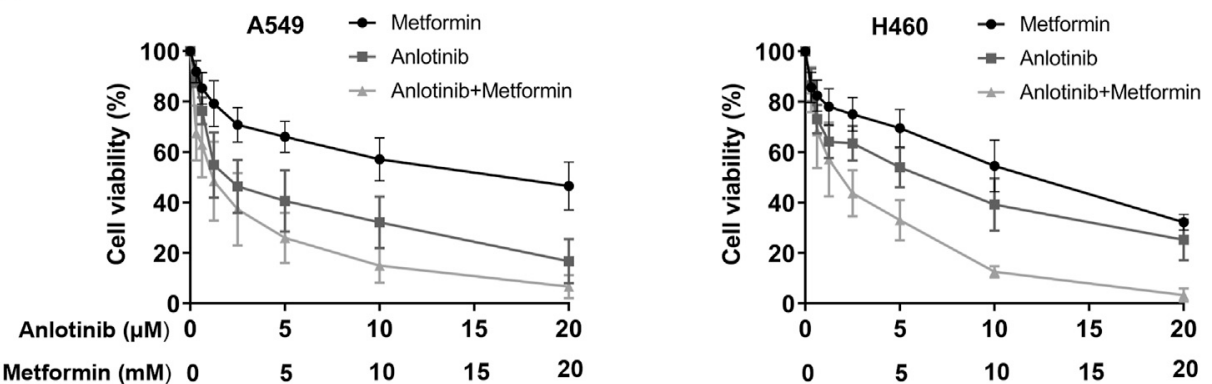

B
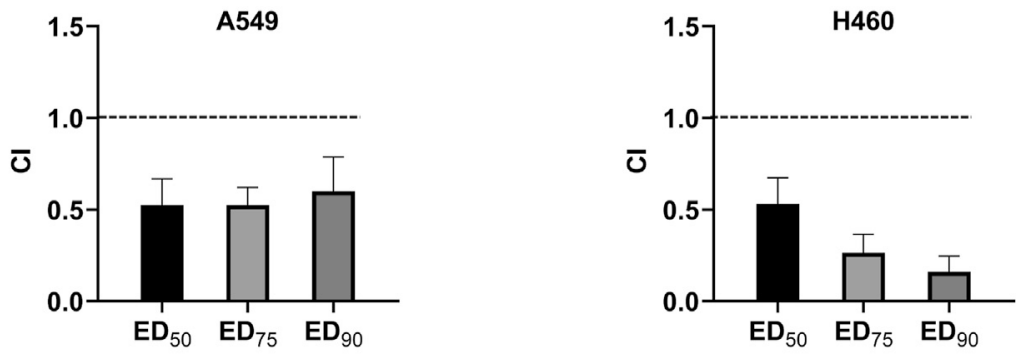

C
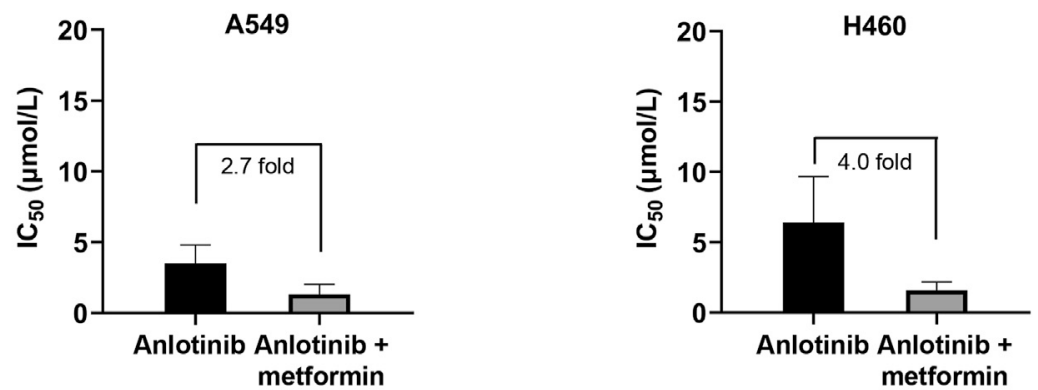

D
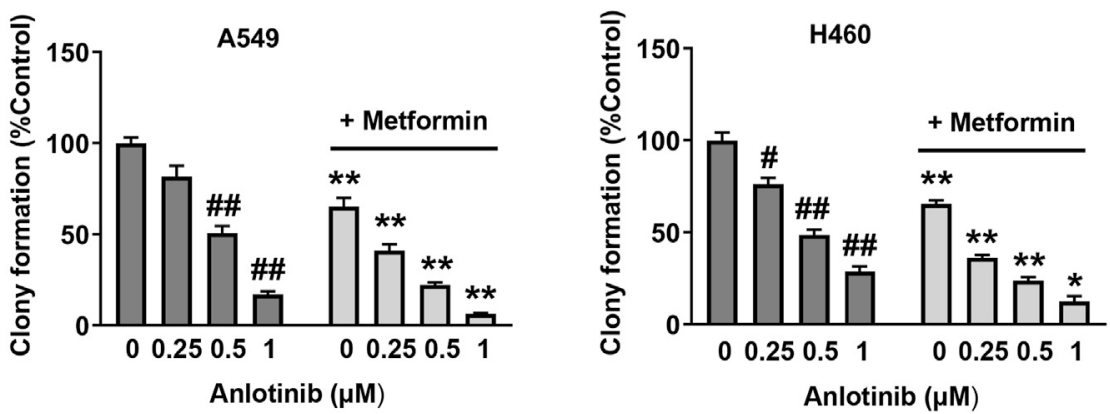

FIGURE 1 | Metformin enhances the cytotoxicity of anlotinib in non-small cell lung cancer (NSCLC) cells (A) A549 and H460 NSCLC cells were treated with anlotinib (range, 0-20 $\mathrm{mmo} / \mathrm{L}$ ), metformin (range, 0-20 mmol/L), or both for 3 days. Cell viability (\%) was determined using the MTT assay (B) The Cl values were determined for effective dose (ED) $E_{50}, E D_{75}$, and $E D_{90}$. Columns represent data from triplicate analyses $\pm S E$ (C) The $I_{50}$ of anlotinib in $A 549$ and $H 460$ cells was reduced by the addition of metformin (D) A549 and H460 cells were exposed to the indicated concentrations of anlotinib alone or anlotinib combined with metformin ( $0.5 \mathrm{mM}$ ) for $48 \mathrm{~h}$. Colony-forming efficiency was determined 10-14 days later. Data are presented as means \pm SE from three independent experiments. Statistical significance was

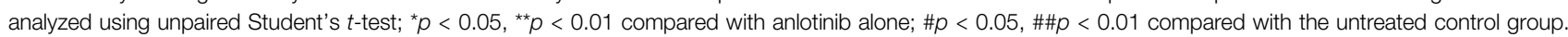



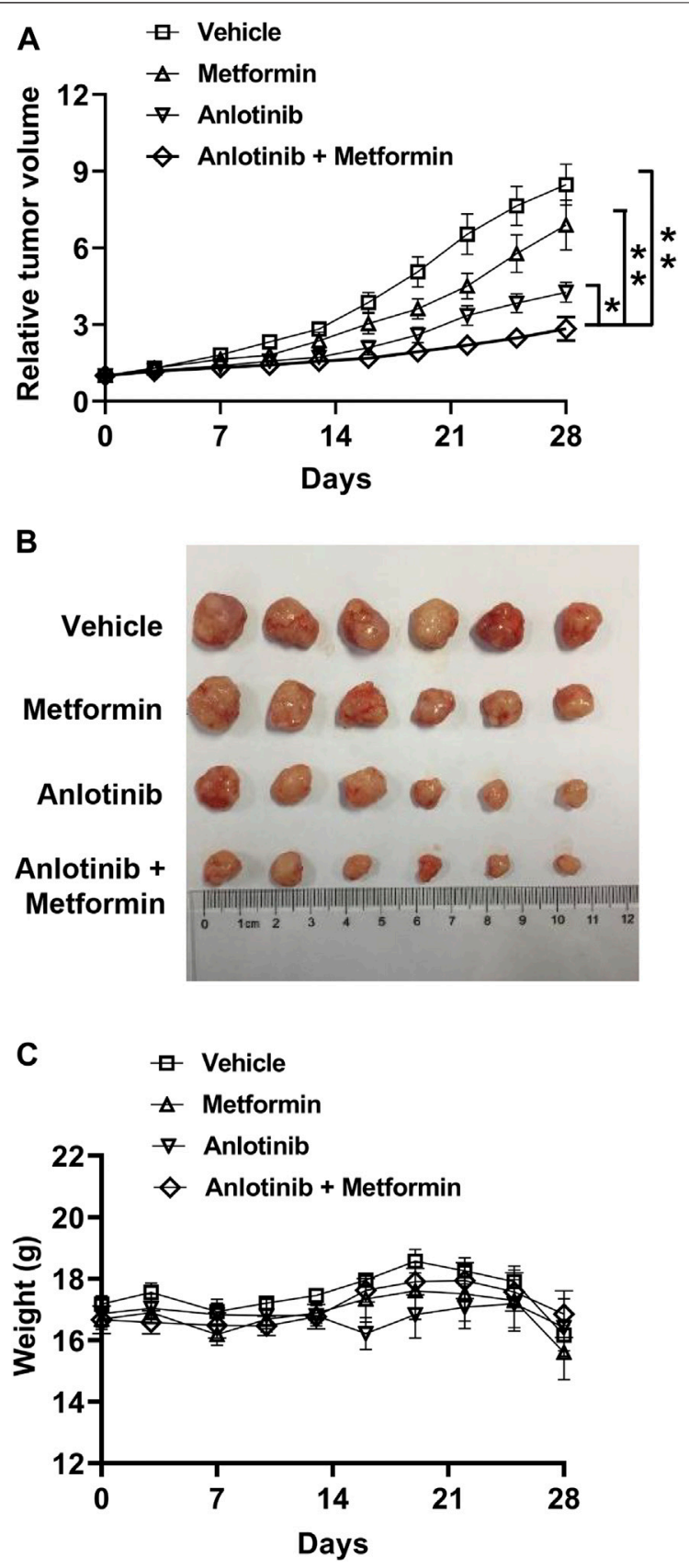

FIGURE 2 | Metformin potentiates the efficacy of anlotinib in a xenograft model (A) Mice with $\mathrm{H} 460$ xenografts were randomly divided into four groups and treated for 28 consecutive days with vehicle (squares), anlotinib (triangles), metformin (inverted triangles), or both anlotinib and metformin (diamonds) as described in the Materials and Methods (B) The sizes of the xenografts at the end of experiment (C) Body weight was measured every 3 days. Data are presented as means \pm SE ( $n=6$ per group). Statistical significance was analyzed using the unpaired Student's $t$-test; ${ }^{\star} p<0.05,{ }^{*} p<0.01$.

\section{Metformin Enhances the Efficacy of Anlotinib in Tumor Xenografts}

We evaluated whether combination treatment with metformin enhanced the antitumor effects of anlotinib using A549 xenografts. Nude mice bearing A549 xenografts were randomized and treated either with anlotinib $(0.75 \mathrm{mg} / \mathrm{kg})$, metformin $(250 \mathrm{mg} / \mathrm{kg})$, or both. Although anlotinib and metformin as monotherapies decreased tumor growth when compared with the control, the combination treatment potentiated the antitumor effects of each single treatment (Figures 2A,B), indicating that the cytotoxicity of anlotinib in the xenograft model was enhanced by the addition of metformin. No significant weight loss was observed in any of the treatment groups, suggesting that the toxicity of the combination was acceptable (Figure 2C).

\section{Anlotinib in Combination with Metformin Induces Cell Apoptosis}

We examined the effects of anlotinib and metformin on cell apoptosis via Hoechst 33342/PI double staining under a fluorescence microscope. As shown in Figures 3A,B, higher numbers of apoptotic and necrotic cells, with condensation of nuclear chromatin and fragmentation, were detected in cells treated with the combination of anlotinib and metformin compared to cells treated with monotherapy. We then determined the expression levels of the apoptosis-related proteins Bcl-2, Bax, caspase 3, and PARP using western blot analysis. The level of Bax protein was substantially increased in response to combination treatment, whereas the expression of $\mathrm{Bcl}-2$ was reduced. We also found that the combination treatment induced caspase 3 and PARP cleavage to an even greater extent (Figures 3C,D). These results suggested that the combination treatment likely induced the Bcl-2/Bax-caspase signaling pathway.

\section{The Synergistic Effect of Anlotinib and Metformin is Mediated by AMPK Activation and mTOR Inhibition}

To clarify the mechanisms underlying the antiproliferative effects of the combination treatment, we studied the effects of the combined treatment on the AMPK and mTOR pathways. Our results showed that anlotinib treatment alone induced phosphorylation of AMPKa at Thr-172. Importantly, AMPK activation increased significantly when anlotinib was combined with metformin (Figure 4). AMPK activation has been shown to reduce cell proliferation, at least in part, by inhibiting mTOR signaling. We found that the combination treatment had a synergistic effect on the suppression of mTOR phosphorylation. It has been reported that acetyl-coA carboxylase (ACC), which plays an important role in the biosynthesis and oxidation of fatty acids, is a downstream substrate of AMPK signaling. Indeed, we found that phosphorylation of ACC at Ser79 was markedly increased when cells were treated with both anlotinib and metformin (Figures 4A,B). Previous studies indicated that metformin might inhibit tumor growth by inhibiting complex I of the respiratory chain and decreasing ATP production. Interestingly, as shown in Figures 4C,D the generation of intracellular ATP in A549 and H460 cells was markedly 


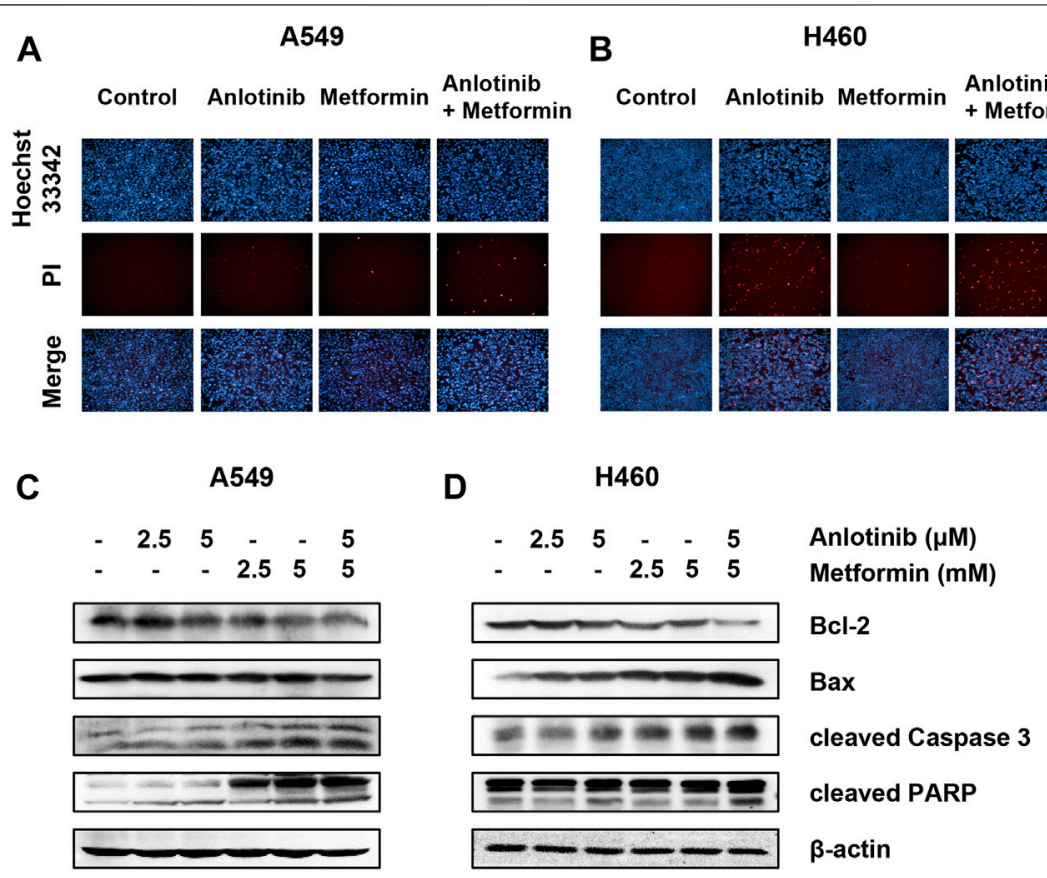

FIGURE 3 | Anlotinib with metformin synergistically induces apoptosis. A549 (A) and H460 (B) cells were incubated with anlotinib (10 $\mu \mathrm{M}$ ), metformin (10 mM), or both for $24 \mathrm{~h}$. After Hoechst 33342 (bright blue color) and PI (red color) double staining, the morphological changes in cells undergoing apoptosis and necrosis were observed under a fluorescence microscope. A549 (C) and H460 (D) cells were treated with the indicated concentrations of anlotinib, metformin, or both for $24 \mathrm{~h}$. Cell lysates were immunoblotted with antibodies against Bcl-2, Bax, cleaved caspase 3, and PARP.

inhibited by exposure to anlotinib in a concentration-dependent manner. In addition, the combination treatment resulted in a lower intracellular ATP levels, suggesting that anlotinib and metformin synergistically inhibited the production of intracellular ATP. HIF-1 transcriptionally promotes anaerobic glycolysis, leading to increased ATP production. Therefore, we next examined HIF-1 $\alpha$ expression in treated cells and found that the expression of HIF-1a was markedly inhibited in both A549 and $\mathrm{H} 460$ cells exposed to the combination treatment under normoxic conditions (Figures 4A,B). These data suggest that the synergistic effect of anlotinib and metformin is related to regulation of the $\mathrm{AMPK} / \mathrm{mTOR}$ signaling pathway.

\section{The Combination of Anlotinib and Metformin Promotes Oxidative Stress}

ROS plays a crucial role in cell apoptosis signaling pathways. Therefore, we examined whether ROS is involved in the cytotoxic effects of anlotinib. The changes in total ROS production were estimated using a cell-permeable probe DCFH-DA. The fluorescence signals for DCF were markedly enhanced in cells treated with anlotinib and metformin compared to the signals in cells treated with anlotinib or metformin alone (Figures $5 \mathbf{A}, \mathbf{B}$ ). Intracellular $\mathrm{NADP}^{+} / \mathrm{NADPH}$ is believed to be a critical redox couple against oxidative stress. Anlotinib significantly increased the ratio of intracellular $\mathrm{NADP}^{+} / \mathrm{NADPH}$ in both cell types. The effect of anlotinib on the $\mathrm{NADP}^{+} / \mathrm{NADPH}$ ratio was potentiated in the presence of metformin, indicating that the combination treatment regulated intracellular redox homeostasis and promoted switching to the oxidative state (Figures 5C,D). These results indicate that the combination treatment induced oxidative stress in A549 and H460 cells.

\section{Anlotinib Combined with Metformin Stimulates the Kinase Activities of p38, JNK, and ERK1/2 Kinases}

ROS play a critical role in cell death via regulation of the mitogenactivated protein kinase (MAPK) family. Here, we studied the effects of anlotinib and metformin both alone and in combination on the kinase activities of ERK1/2, p38, and JNK. Our results showed that the combination treatment exerted a synergistic effect on p38, JNK, and ERK1/2 activation (Figure 6). Thus, these results indicate that anlotinib in combination with metformin enhances the phosphorylation of p38, JNK, and ERK1/2, which may be mediated by ROS.

\section{DISCUSSION}

The anticancer effect of anlotinib has been reported to be associated with its function as a multikinase inhibitor in angiogenic signaling pathways. Anlotinibalso suppresses tumor growth by blocking c-Kit, RET, Aurora-B, c-FMS, and DDR1 
A

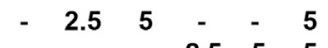

$-\quad-\quad-2.5 \quad 55$

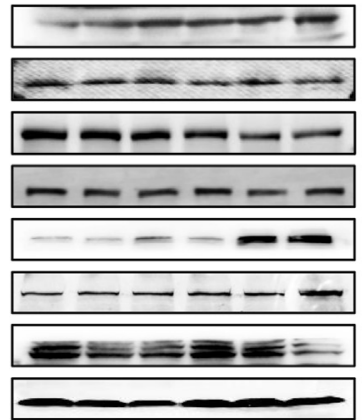

C

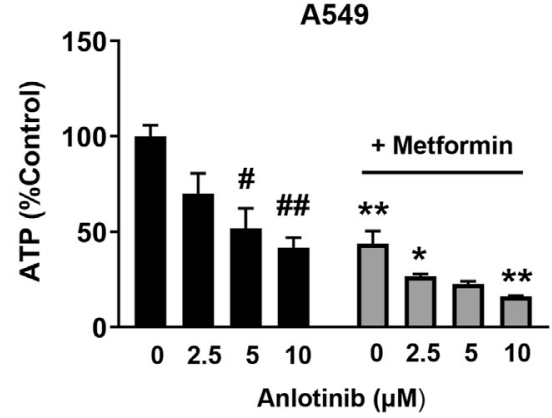

B

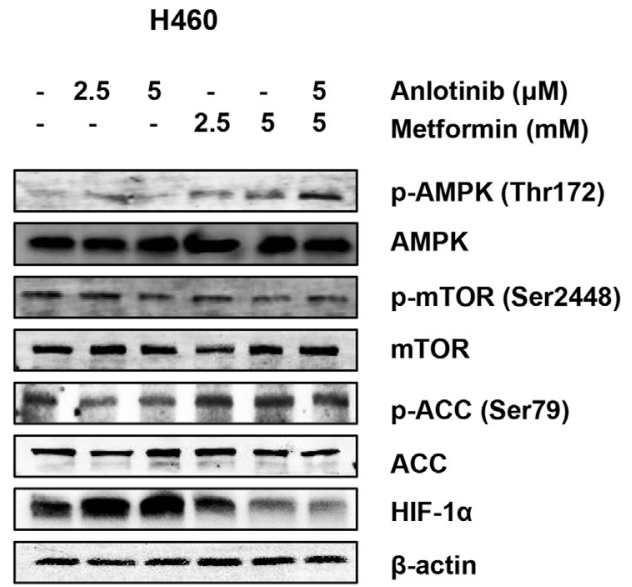

D

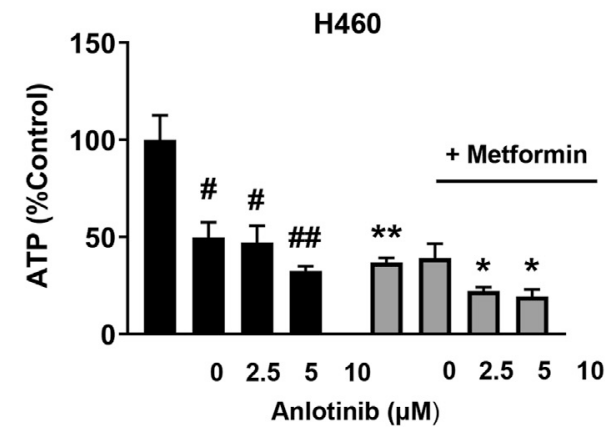

FIGURE 4 | Anlotinib in combination with metformin promotes the activation of AMPK and inhibition of mTOR. A549 (A) and H460 (B) cells were treated with the indicated concentrations of anlotinib, metformin, or both for $24 \mathrm{~h}$. Cell lysates were harvested and immunoblotted with the indicated antibodies. A549 (C) and H460 (D) were exposed to the indicated concentrations of anlotinib alone or anlotinib and metformin (5 mM) for 24 h. Relative intracellular ATP levels were determined using an assay kit according to the manufacturer's instructions. Data are presented as means \pm SE from three independent experiments. Statistical significance was analyzed using unpaired Student's $t$-test; ${ }^{\star} p<0.05,{ }^{\star \star} p<0.01$ compared with anlotinib alone; \#p $<0.05$, \#\#p $<0.01$ compared with the untreated control group.

(Sun et al., 2016). In this study, we revealed that metformin could augment the cytotoxic effects of anlotinib. The enhanced synergistic effect of anlotinib and metformin inhibited the proliferation of NSCLC cells both in vitro and in vivo. The concentrations of anlotinib and metformin administrated in our in vitro and in vivo experiments are equal to concentrations administrated in previous studies (Chen et al., 2012; Xie et al., 2020), though higher than the therapeutic doses in humans. Low concentrations of metformin (typically 0.1-0. $3 \mathrm{mM}$ ) selectively inhibited cancer stem cells, but these low doses had little effect on the proliferation of cancer cells (Ben et al., 2010). The combination treatment increased PARP1 cleavage, caspase- 3 cleavage, and the $\mathrm{Bax} / \mathrm{Bcl}-2$ ratio, suggesting that the combination treatment triggered apoptosis, possibly mediated by the mitochondrial-dependent pathway.

Previous studies have mostly focused on the effects of anlotinib on angiogenesis and proliferation (Song F. et al., 2020). Comparatively, little attention has been paid to the effects of anlotinib on energy metabolism. In this study, we found that anlotinib significantly decreased the ATP content in NSCLC cells. It is known that decreased ATP can activate
AMPK, which inhibits growth by blocking the mTOR pathway (Faubert et al., 2015). Metformin has been shown to reduce cell proliferation through the activation of AMPK and inactivation of mTOR signaling (Rocha et al., 2011; Storozhuk et al., 2013). In our study, we found that anlotinib activated AMPK, downregulated mTOR phosphorylation, and induced apoptosis. Importantly, metformin, as an indirect AMPK activator, potentiates the effects of anlotinib on the AMPK and mTOR signaling pathways. In agreement with our findings, Groenendijk et al. showed that sorafenib synergizes with metformin in NSCLC through the activation of the AMPK pathway (Groenendijk et al., 2015).

As signaling molecules, ROS play a crucial role in cell death signal transduction pathways. Excessive ROS can cause damage to biomacromolecules and promote autophagy, apoptosis, or necrosis (Wong et al., 2010). The $\mathrm{NADP}^{+}$/ $\mathrm{NADPH}$ redox couple is involved in buffering ROS and sustaining antioxidant defenses (Aon et al., 2010). Recently, Yang et al. reported that anlotinib can directly inhibit the proliferation of and induce apoptosis in pancreatic cancer cells through ROS-activated ER stress via PERK/p-eIF2a/ATF4 
A

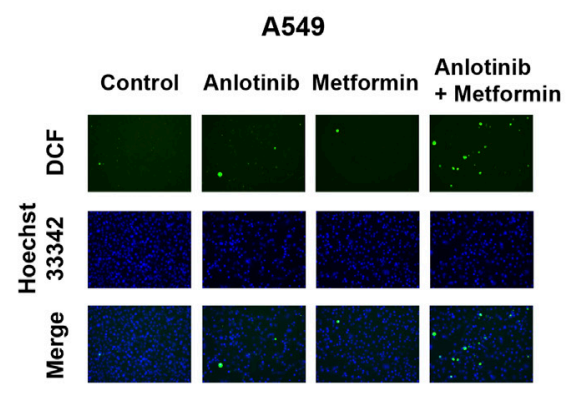

C

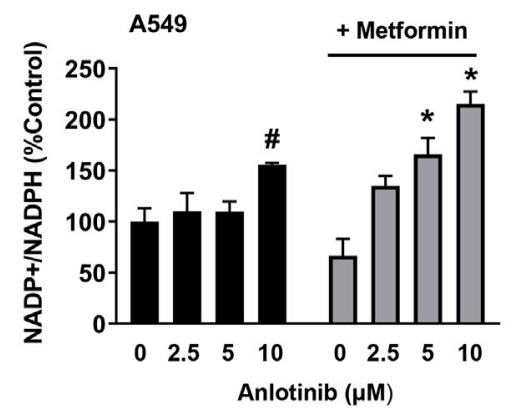

B

H460

Control Anlotinib Metformin $\begin{aligned} & \text { Anlotinib } \\ & + \text { Metformin }\end{aligned}$
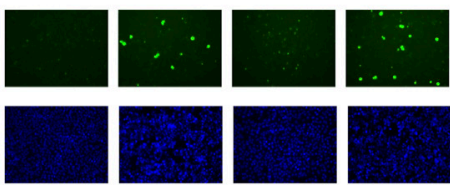

Dan

(20)

$+2$

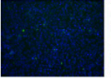

$\therefore \therefore$
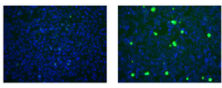

D

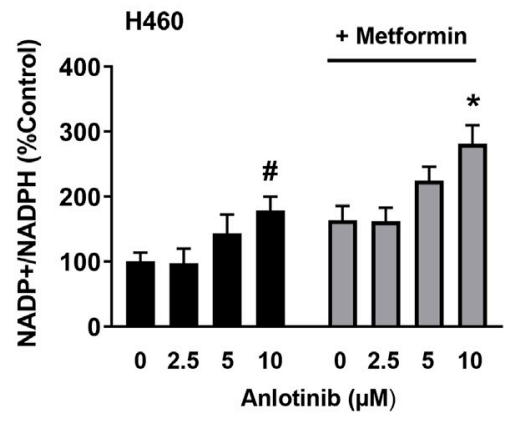

FIGURE 5 | Anlotinib combined with metformin increased intracellular ROS levels and the NADP ${ }^{+}$NADPH ratio. A549 (A) and H460 (B) cells were incubated with anlotinib $(5 \mu \mathrm{M})$, metformin $(5 \mathrm{mM})$, or both for $24 \mathrm{~h}$. After DCF (green color) and Hoechst 33342 (bright blue color) double staining, cellular DCF fluorescence was observed under a fluorescence microscope. A549 (C) and H460 (D) cells were exposed to the indicated concentrations of anlotinib or anlotinib and metformin (5 mM) for $24 \mathrm{~h}$. The $\mathrm{NADP}^{+} / \mathrm{NADPH}$ ratio was measured in A549 and $\mathrm{H} 460$ cells using an assay kit, as described in the Materials and Methods. Data are presented as means \pm SE from three independent experiments. Statistical significance was analyzed using unpaired Student's $t$-test; ${ }^{*} p<0.05$ compared with anlotinib alone; \#p $<$ 0.05 compared with the untreated control group.

A

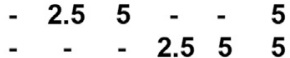

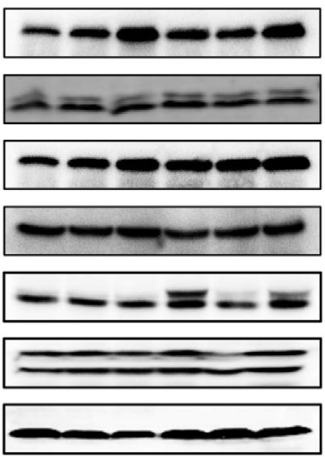

B

H460
Anlotinib $(\mu \mathrm{M})$

Metformin $(\mathrm{mM})$

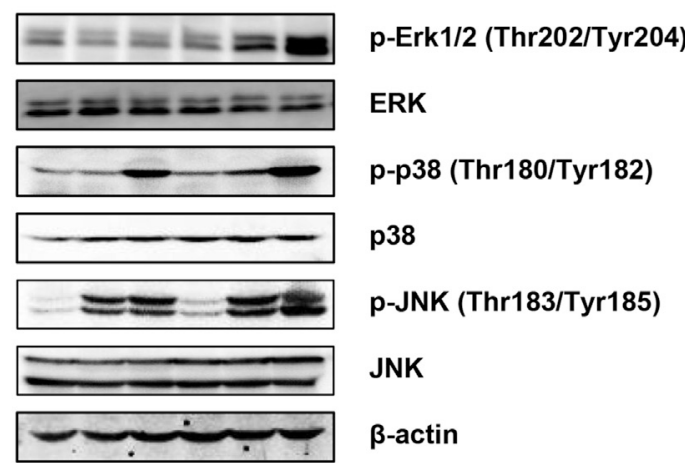

FIGURE 6 | Anlotinib combined with metformin increases the phosphorylation of ERK1/2, p38, and JNK. A549 (A) and H460 (B) cells were incubated with the indicated concentrations of anlotinib, metformin, or both for $24 \mathrm{~h}$. Cell lysates were immunoblotted with antibodies against pERK1/2 and ERK1/2, p-p38 and p38, and pJNK and JNK. 
(Yang L. et al., 2020). Similarly, we found that anlotinib or metformin increased ROS production and the $\mathrm{NADP}^{+}$/ $\mathrm{NADPH}$ ratio in NSCLC cells, indicating that anlotinib or metformin can disrupt intracellular redox homeostasis and induce oxidative stress. Moreover, the combination treatment stimulated ROS generation to an even greater extent. Growing evidence has shown that members of the MAPK family, including p38 MAPK, JNK, and ERK, are critically involved inthe oxidative stress response (El-Najjar et al., 2010). The p38 MAPK and JNK pathways are related to apoptosis, yet activation of ERK is also related to cell survival (Zhang et al., 2019). However, many studies have shown that activation of ERK can promote cell death via apoptotic pathways and cell cycle arrest (Wang et al., 2000; Tang et al., 2002; Song Y. et al., 2020). These effects require sustained activation of ERK in specific subcellular compartments (Wang et al., 2000). In our study, we observed that anlotinib increased the phosphorylation of ERK1/2, p38, and JNK, and these increases in phosphorylation were greatest when cells were treated with both anlotinib and metformin. These data suggest that anlotinib-induced apoptosis may be the result of elevated intracellular ROS, which may function as upstream regulators of the $\mathrm{p} 38 / \mathrm{JNK}$ MAPK and ERK pathways. In contrast, other studies have shown that anlotinib attenuates ERK activation in diverse cancer cells (Yang Q. et al., 2020; Hu et al., 2020; Lian et al., 2020). Cagnol et al. reported that ERK activity depends on the presence of ROS (Cagnol and Chambard, 2010). Differences in intracellular ROS levels and patterns of ROS accumulation may contribute to this inconsistency.

Thus, several mechanisms may contribute to the synergistic anticancer effect of anlotinib and metformin. The first involves decreased ATP-induced AMPK activation and mTOR inhibition. Additionally, ROS-mediated induction of p38/JNK MAPK and ERK signaling may be involved.

\section{REFERENCES}

Alimova, I. N., Liu, B., Fan, Z., Edgerton, S. M., Dillon, T., Lind, S. E., et al. (2009). Metformin Inhibits Breast Cancer Cell Growth, colony Formation and Induces Cell Cycle Arrest In Vitro. Cell Cycle 8 (6), 909-915. doi:10.4161/cc.8.6.7933

Aon, M. A., Cortassa, S., and O'Rourke, B. (2010). Redox-optimized ROS Balance: a Unifying Hypothesis. Biochim. Biophys. Acta (Bba) - Bioenerg. 1797 (6-7), 865-877. doi:10.1016/j.bbabio.2010.02.016

Assi, H. I., Kamphorst, A. O., Moukalled, N. M., and Ramalingam, S. S. (2018). Immune Checkpoint Inhibitors in Advanced Non-small Cell Lung Cancer. Cancer 124 (2), 248-261. doi:10.1002/cncr.31105

Ben Sahra, I., Le Marchand-Brustel, Y., Tanti, J. F., and Bost, F. (2010). Metformin in Cancer Therapy: a New Perspective for an Old Antidiabetic Drug?. Mol. Cancer Ther. 9 (5), 1092-1099. doi:10.1158/1535-7163.MCT-09-1186

Bray, F., Ferlay, J., Soerjomataram, I., Siegel, R. L., Torre, L. A., and Jemal, A. (2018). Global Cancer Statistics 2018: GLOBOCAN Estimates of Incidence and Mortality Worldwide for 36 Cancers in 185 Countries. CA: A Cancer J. Clinicians 68 (6), 394-424. doi:10.3322/caac.21492

Cagnol, S., and Chambard, J. C. (2010). ERK and Cell Death: Mechanisms of ERKInduced Cell Death - Apoptosis, Autophagy and Senescence. FEBS J. 277 (1), 2-21. doi:10.1111/j.1742-4658.2009.07366.x

Chen, G., Xu, S., Renko, K., and Derwahl, M. (2012). Metformin Inhibits Growth of Thyroid Carcinoma Cells, Suppresses Self-Renewal of Derived Cancer Stem

\section{CONCLUSION}

Metformin increases the sensitivity of NSCLC cells to anlotinib both in vitro and in vivo, providing a potential rationale for the combination of anlotinib with metformin for patients with NSCLC or other cancers.

\section{DATA AVAILABILITY STATEMENT}

The original contributions presented in the study are included in the article/supplementary materials, further inquiries can be directed to the corresponding author.

\section{ETHICS STATEMENT}

The animal study was reviewed and approved by Laboratory Animal Ethics Committee of Tianjin Medical University Cancer Institute and Hospital.

\section{AUTHOR CONTRIBUTIONS}

$\mathrm{ZZ}$ and ZY designed the research. TJ, HS and SX performed the experiments and collected data. ZZ, TJ, SX and CZ conducted data analysis. ZZ wrote the paper. ZY and GY revised the paper. All authors reviewed the manuscript.

\section{FUNDING}

This work was supported by the National Natural Science Foundation of China (No. 81402481) and Tianjin Science and Technology Plan Project (No. 18ZXXYSY00070).

Cells, and Potentiates the Effect of Chemotherapeutic Agents. J. Clin. Endocrinol. Metab. 97 (4), E510-E520. doi:10.1210/jc.2011-1754

Chi, Y., Fang, Z., Hong, X., Yao, Y., Sun, P., Wang, G., et al. (2018). Safety and Efficacy of Anlotinib, a Multikinase Angiogenesis Inhibitor, in Patients with Refractory Metastatic Soft-Tissue Sarcoma. Clin. Cancer Res. 24, 5233-5238. doi:10.1158/1078-0432.CCR-17-3766

Deng, J., Peng, M., Wang, Z., Zhou, S., Xiao, D., Deng, J., et al. (2019). Novel Application of Metformin Combined with Targeted Drugs on Anticancer Treatment. Cancer Sci. 110 (1), 23-30. doi:10.1111/cas.13849

Dowling, R. J., Goodwin, P. J., and Stambolic, V. (2011). Understanding the Benefit of Metformin Use in Cancer Treatment. BMC Med. 9, 33. doi:10.1186/17417015-9-33

El-Najjar, N., Chatila, M., Moukadem, H., Vuorela, H., Ocker, M., Gandesiri, M., et al. (2010). Reactive Oxygen Species Mediate Thymoquinone-Induced Apoptosis and Activate ERK and JNK Signaling. Apoptosis 15 (2), 183-195. doi:10.1007/s10495-009-0421-z

Faubert, B., Vincent, E. E., Poffenberger, M. C., and Jones, R. G. (2015). The AMPActivated Protein Kinase (AMPK) and Cancer: Many Faces of a Metabolic Regulator. Cancer Lett. 356 (2), 165-170. doi:10.1016/j.canlet.2014.01.018

Fontaine, E. (2018). Metformin-Induced Mitochondrial Complex I Inhibition: Facts, Uncertainties, and Consequences. Front. Endocrinol. 9, 753. doi:10.3389/ fendo.2018.00753

Gao, Y., Liu, P., and Shi, R. (2020). Anlotinib as a Molecular Targeted Therapy for Tumors (Review). Oncol. Lett. 20 (2), 1001-1014. doi:10.3892/ol.2020.11685 
Groenendijk, F. H., Mellema, W. W., van der Burg, E., Schut, E., Hauptmann, M., Horlings, H. M., et al. (2015). Sorafenib Synergizes with Metformin in NSCLC through AMPK Pathway Activation. Int. J. Cancer 136 (6), 1434-1444. doi:10.1002/ijc.29113

Han, B., Li, K., Wang, Q., Zhang, L., Shi, J., Wang, Z., et al. (2018). Effect of Anlotinib as a Third-Line or Further Treatment on Overall Survival of Patients with Advanced Non-small Cell Lung Cancer. JAMA Oncol. 4 (11), 1569. doi:10.1001/jamaoncol.2018.3039

Hirsch, F. R., Scagliotti, G. V., Mulshine, J. L., Kwon, R., Curran, W. J., Wu, Y.-L., et al. (2017). Lung Cancer: Current Therapies and New Targeted Treatments. The Lancet 389 (10066), 299-311. doi:10.1016/S0140-6736(16)30958-8

Hu, H., Liu, Y., Tan, S., Xie, X. X., He, J., Luo, F., et al. (2020). Anlotinib Exerts Anti-cancer Effects on KRAS-Mutated Lung Cancer Cell through Suppressing the MEK/ERK Pathway. Cmar 12, 3579-3587. doi:10.2147/CMAR.S243660

Huang, J., Xiao, J., Fang, W., Lu, P., Fan, Q., Shu, Y., et al. (2021). Anlotinib for Previously Treated Advanced or Metastatic Esophageal Squamous Cell Carcinoma: A Double-blind Randomized Phase 2 Trial. Cancer Med. 10 (5), 1681-1689. doi:10.1002/cam4.3771

Kalender, A., Selvaraj, A., Kim, S. Y., Gulati, P., Brûlé, S., Viollet, B., et al. (2010). Metformin, Independent of AMPK, Inhibits mTORC1 in a Rag GTPasedependent Manner. Cel. Metab. 11 (5), 390-401. doi:10.1016/ j.cmet.2010.03.014

Khawaja, M. R., Nick, A. M., Madhusudanannair, V., Fu, S., Hong, D., Mcquinn, L. M., et al. (2016). Phase I Dose Escalation Study of Temsirolimus in Combination with Metformin in Patients with Advanced/refractory Cancers. Cancer Chemother. Pharmacol. 77 (5), 973-977. doi:10.1007/s00280-0163009-7

Lian, Z., Du, W., Zhang, Y., Fu, Y., Liu, T., Wang, A., et al. (2020). Anlotinib Can Overcome Acquired Resistance to EGFR-TKIs via FGFR1 Signaling in Nonsmall Cell Lung Cancer without Harboring EGFR T790M Mutation. Thorac. Cancer 11 (7), 1934-1943. doi:10.1111/1759-7714.13485

Ma, J., Song, Y., Shou, J., Bai, Y., Li, H., Xie, X., et al. (2020). Anlotinib for Patients with Metastatic Renal Cell Carcinoma Previously Treated with One Vascular Endothelial Growth Factor Receptor-Tyrosine Kinase Inhibitor: A Phase 2 Trial. Front. Oncol. 10, 664. doi:10.3389/fonc.2020.00664

Martin-Castillo, B., Pernas, S., Dorca, J., Álvarez, I., Martínez, S., Pérez-Garcia, J. M., et al. (2018). A Phase 2 Trial of Neoadjuvant Metformin in Combination with Trastuzumab and Chemotherapy in Women with Early HER2-Positive Breast Cancer: the METTEN Study. Oncotarget 9 (86), 35687-35704. doi:10.18632/oncotarget.26286

Rocha, G. Z., Dias, M. M., Ropelle, E. R., Osório-Costa, F., Rossato, F. A., Vercesi, A. E., et al. (2011). Metformin Amplifies Chemotherapy-Induced AMPK Activation and Antitumoral Growth. Clin. Cancer Res. 17 (12), 3993-4005. doi:10.1158/1078-0432.CCR-10-2243

Shen, G., Zheng, F., Ren, D., Du, F., Dong, Q., Wang, Z., et al. (2018). Anlotinib: a Novel Multi-Targeting Tyrosine Kinase Inhibitor in Clinical Development. J. Hematol. Oncol. 11 (1), 120. doi:10.1186/s13045-018-0664-7

Song, F., Hu, B., Cheng, J.-W., Sun, Y.-F., Zhou, K.-Q., Wang, P.-X., et al. (2020a). Anlotinib Suppresses Tumor Progression via Blocking the VEGFR2/PI3K/AKT cascade in Intrahepatic Cholangiocarcinoma. Cell Death Dis 11 (7), 573. doi:10.1038/s41419-020-02749-7

Song, Y., Sun, X., Duan, F., He, C., Wu, J., Huang, X., et al. (2020b). SYPL1 Inhibits Apoptosis in Pancreatic Ductal Adenocarcinoma via Suppression of ROSInduced ERK Activation. Front. Oncol. 10, 1482. doi:10.3389/fonc.2020.01482

Storozhuk, Y., Hopmans, S. N., Sanli, T., Barron, C., Tsiani, E., Cutz, J.-C., et al. (2013). Metformin Inhibits Growth and Enhances Radiation Response of Nonsmall Cell Lung Cancer (NSCLC) through ATM and AMPK. Br. J. Cancer 108 (10), 2021-2032. doi:10.1038/bjc.2013.187

Sun, Y., Du, F., Gao, M., Ji, Q., Li, Z., Zhang, Y., et al. (2018). Anlotinib for the Treatment of Patients with Locally Advanced or Metastatic Medullary Thyroid Cancer. Thyroid 28 (11), 1455-1461. doi:10.1089/thy.2018.0022

Sun, Y., Niu, W., Du, F., Du, C., Li, S., Wang, J., et al. (2016). Safety, Pharmacokinetics, and Antitumor Properties of Anlotinib, an Oral
Multi-Target Tyrosine Kinase Inhibitor, in Patients with Advanced Refractory Solid Tumors. J. Hematol. Oncol. 9 (1), 105. doi:10.1186/s13045016-0332-8

Syed, Y. Y. (2018). Anlotinib: First Global Approval. Drugs 78 (10), 1057-1062. doi:10.1007/s40265-018-0939-x

Tang, D., Wu, D., Hirao, A., Lahti, J. M., Liu, L., Mazza, B., et al. (2002). ERK Activation Mediates Cell Cycle Arrest and Apoptosis after DNA Damage Independently of P53. J. Biol. Chem. 277 (15), 12710-12717. doi:10.1074/ jbc.M111598200

Wang, X., Martindale, J. L., and Holbrook, N. J. (2000). Requirement for ERK Activation in Cisplatin-Induced Apoptosis. J. Biol. Chem. 275 (50), 39435-39443. doi:10.1074/jbc.M004583200

Wheaton, W. W., Weinberg, S. E., Hamanaka, R. B., Soberanes, S., Sullivan, L. B., Anso, E., et al. (2014). Metformin Inhibits Mitochondrial Complex I of Cancer Cells to Reduce Tumorigenesis. eLife 3. doi:10.7554/eLife.02242

Wong, C. H., Iskandar, K. B., Yadav, S. K., Hirpara, J. L., Loh, T., and Pervaiz, S. (2010). Simultaneous Induction of Non-canonical Autophagy and Apoptosis in Cancer Cells by ROS-dependent ERK and JNK Activation. PLoS One 5 (4), e9996. doi:10.1371/journal.pone.0009996

Wu, D., Nie, J., Hu, W., Dai, L., Zhang, J., Chen, X., et al. (2020). A Phase II Study of Anlotinib in 45 Patients with Relapsed Small Cell Lung Cancer. Int. J. Cancer 147, 3453-3460. doi:10.1002/ijc.33161

Xie, J., Ye, J., Cai, Z., Luo, Y., Zhu, X., Deng, Y., et al. (2020). GPD1 Enhances the Anticancer Effects of Metformin by Synergistically Increasing Total Cellular Glycerol-3-Phosphate. Cancer Res. 80 (11), 2150-2162. doi:10.1158/00085472.CAN-19-2852

Yang, L., Zhou, X., Sun, J., Lei, Q., Wang, Q., Pan, D., et al. (2020a). Reactive Oxygen Species Mediate Anlotinib-Induced Apoptosis via Activation of Endoplasmic Reticulum Stress in Pancreatic Cancer. Cel. Death Dis. 11 (9), 766. doi:10.1038/s41419-020-02938-4

Yang, Q., Ni, L., Imani, S., Xiang, Z., Hai, R., Ding, R., et al. (2020b). Anlotinib Suppresses Colorectal Cancer Proliferation and Angiogenesis via Inhibition of AKT/ERK Signaling Cascade. Cmar 12, 4937-4948. doi:10.2147/ CMAR.S252181

Zhang, G., He, J., Ye, X., Zhu, J., Hu, X., Shen, M., et al. (2019). $\beta$-Thujaplicin Induces Autophagic Cell Death, Apoptosis, and Cell Cycle Arrest through ROSMediated Akt and P38/ERK MAPK Signaling in Human Hepatocellular Carcinoma. Cel. Death Dis. 10 (4), 255. doi:10.1038/s41419-019-1492-6

Zhang, H. H., and Guo, X. L. (2016). Combinational Strategies of Metformin and Chemotherapy in Cancers. Cancer Chemother. Pharmacol. 78 (1), 13-26. doi:10.1007/s00280-016-3037-3

Zhou, A. P., Bai, Y., Song, Y., Luo, H., Ren, X. B., Wang, X., et al. (2019). Anlotinib versus Sunitinib as First-Line Treatment for Metastatic Renal Cell Carcinoma: A Randomized Phase II Clinical Trial. Oncol. 24 (8), e702-e708. doi:10.1634/ theoncologist.2018-0839

Conflict of Interest: The authors declare that the research was conducted in the absence of any commercial or financial relationships that could be construed as a potential conflict of interest.

Publisher's Note: All claims expressed in this article are solely those of the authors and do not necessarily represent those of their affiliated organizations, or those of the publisher, the editors and the reviewers. Any product that may be evaluated in this article, or claim that may be made by its manufacturer, is not guaranteed or endorsed by the publisher.

Copyright $\odot 2021$ Zhu, Jiang, Suo, Xu, Zhang, Ying and Yan. This is an open-access article distributed under the terms of the Creative Commons Attribution License (CC $B Y$ ). The use, distribution or reproduction in other forums is permitted, provided the original author(s) and the copyright owner(s) are credited and that the original publication in this journal is cited, in accordance with accepted academic practice. No use, distribution or reproduction is permitted which does not comply with these terms. 